\title{
Efficiency of Using Tuned Mass Damper to Reduce Damage after Strong Earthquakes
}

\author{
Andrei Benin ${ }^{1 *}$, Alexander Uzdin $^{1}$ and Olga Nesterova ${ }^{1}$ \\ ${ }^{1}$ Emperor Alexander I St. Petersburg State Transport University, 190031, 9 Moskovsky pr., St. \\ Petersburg, Russia
}

\begin{abstract}
The efficiency of applying tuned mass damper is substantiated for reducing the damageability of structures under strong earthquakes. Two models of structure damage accumulation are considered. The first model is elastoplastic one, the damage degree of the model being determined by the work of plastic deformation forces. The second model is a model with degrading rigidity the damage degree of the model is connected with the development of cracks and is determined by the maximum displacement of the structure in its loading history. For the first type of nonlinearity, i.e. the first model there is an amplitude-frequency characteristic and the optimum tuning of the mass damper corresponds to the maximum of this characteristic. For the second model of accumulation of damages there is no frequency response, therefore the mass damper tuning obtained with harmonic action on the elastic system was used. Calculations of the system with mass damper and without it using earthquake accelerograms have been carried out. Accelerograms, the most unfavorable in terms of the spectral composition for the structures under consideration, were chosen taking into account peak accelerations and energy characteristics of the seismic action. It has been established for elastoplastic systems that tuned mass damper reduces the time of the structure being in plastic stage and the work of plastic deformation forces. For systems with degrading stiffness tuned mass damper reduces the maximum movement of the system in its loading history.
\end{abstract}

\section{Introduction}

Tuned mass dampers (MD) have been used in earthquake engineering since the second half of the last century. For example in the former USSR a television tower in Alma-Ata, designed under the direction of B.G. Korenev, was constructed using the MD. Theoretical studies the MD of seismic oscillations were carried out in the USSR by B.G. Korenev and L.M. Reznikov [1], V.S. Polyakov[2], A.M. Uzdin and A.A. Nikitin[3] and other experts. In the last 30 years by foreign experts also studied the MD performance. It has been established that it is necessary to take damping into account when selecting the MD parameters $[4,5]$. At the beginning, the MD was applied in high-rise construction to reduce wind loads $[6,7]$. However, a number of high-rise buildings with the MD, for example, in

\footnotetext{
*Corresponding author: benin.andrey@mail.ru
} 
Guangzhou and Taiwan, were erected in the earthquake prone areas to reduce both wind and seismic loads. Serious successes were achieved in Armenia under the leadership of M. Melkumyan in designing and constructing buildings with a flexible upper floor, acting as the MD.

The abovementioned developments deal with the MD tuning for harmonic or random actions and an elastic system behavior. In these investigations the maximum acceleration or displacements of the protected system were regarded as a criterion of the MD efficiency. For buildings under strong earthquakes an accumulation of damages, a change of the eigen oscillation period and damping of the main system take place. In this case, the initial assumptions of the MD analysis and the criteria of its effectiveness used in the abovementioned studies are not valid. The analysis of the behavior of structures with degrading stiffness in the MD presence has been performed by A.A. Nikitin and described in the monograph [8]. It included the calculation of a system with the degrading rigidity equipped by the MD under the actions of Helen and Gazli earthquakes. However, the choice of actions was random. In connection with the foregoing, the problem of investigating the behavior of structures with the MD requires additional studies.

\section{Methods of research}

The research methods included choosing the model of structure nonlinearity during the damage accumulation, the selecting the MD parameters, selecting dangerous accelerograms, and the numerical modeling of seismic oscillations of systems with and without the MD. To describe the nonlinear structure behavior, two models of damage accumulation in the structure are considered. The first model of damage accumulation is elastically-plastic one. The system deformation diagram is a Prandtl diagram. The work of plastic deformation forces is taken as an index of the structure damageability. Structures with such a diagram are characterized by the amplitude-frequency characteristic (AFC). the MD tuning can be selected basing on the maximum position on the AFC.

To select a dangerous accelerogram, the authors proposed to build the spectra of plastic deformation work. For this purpose, a reaction to a given accelerogram of an oscillator connected to the base by a dry friction damper, which has a Prandtl diagram as its deformation diagram, was considered. Consequently one can get the dependence of plastic deformation force work on the initial eigen oscillation structure period and structure elastic force. This indicator of earthquake intensity, proposed by the authors in [9], is very useful for analysing elastoplastic systems.

Fig. 1 shows examples of the spectra of plastic force work for the Foshan (China) earthquake with the peak ground acceleration (PGA) equal to $9.5 \mathrm{~m} / \mathrm{s}^{2}$, and Gazli (Uzbekistan) earthquake with the peak ground acceleration PGA $=13.2 \mathrm{~m} / \mathrm{s}^{2}$. The first accelerogram is more dangerous because it has the greatest value of the work of plastic deformation forces for a given initial eigen period $(\mathrm{T}=0.464 \mathrm{sec})$ and for the elastic limit of the structure ( $30 \%$ of structure weight), although the PGA of the second accelerogram is bigger than that of the first one. The structure with the given the MD and the selected accelerogram was calculated in a standard manner according to the program developed by the authors using the Duhamel integral. 
a)

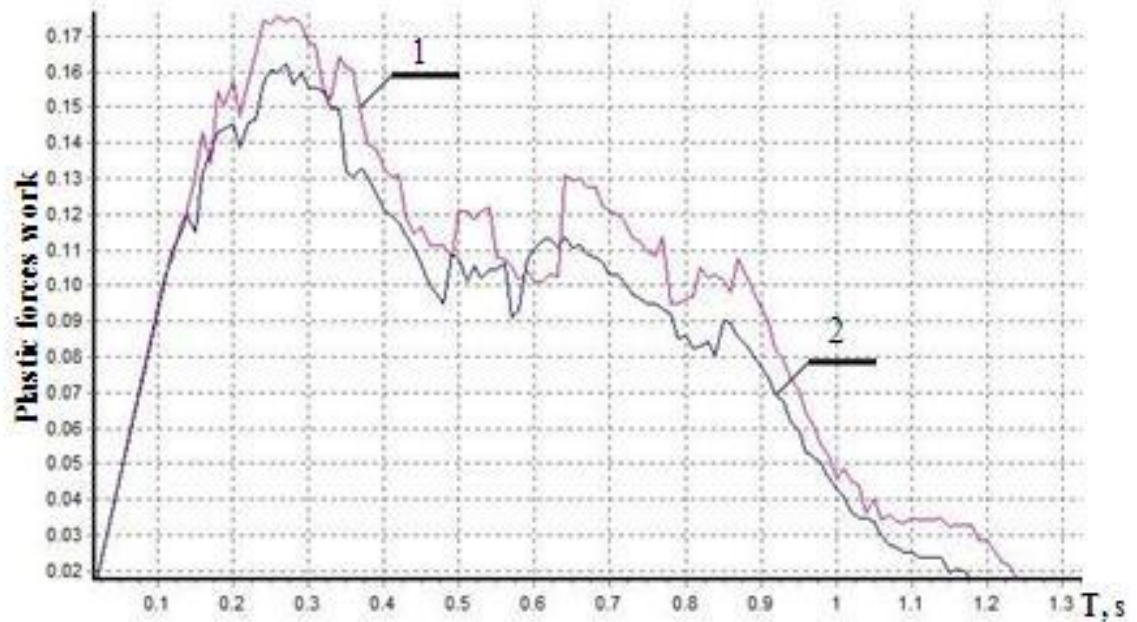

b)

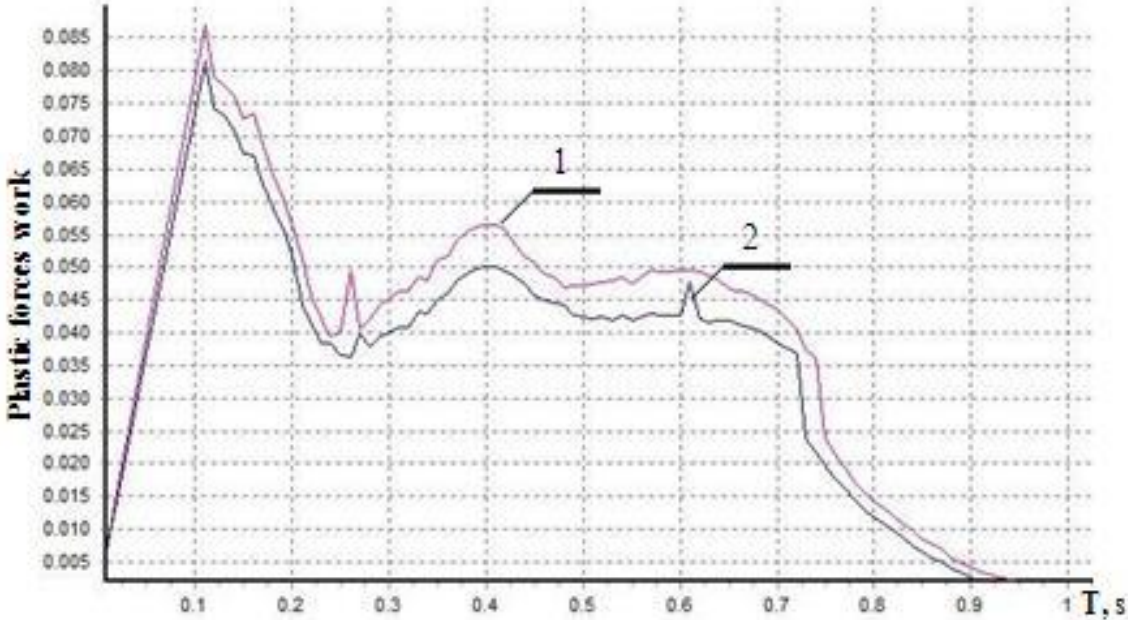

Fig. 1 Spectra of plastic deformation force work for earthquakes Foshan (a) and Gazli (b) 1 - for damping equal to $7.5 \%$ of critical value, 2 - - for damping equal to $10 \%$ of critical value

The second model is a model with degrading rigidity caused by damage accumulation and is typical for brittle materials. For a system with degrading rigidity the KirikovAmankulov model [10] was used as the basis in taking into account the nonlinearity of the structure behavior. This model was considered in [10] in detail.

In this model the system rigidity $\mathrm{R}$ decreases, and the damping and nonlinearity parameter $\kappa$ increase linearly with the increase of the displacements maximum in system loading history. As long as the value of $\mathrm{u}$ is smaller than the elastic limit $\mathrm{u}_{\mathrm{el}}$, the rigidity of the system is constant. After $\mathrm{u}$ becomes bigger than $\mathrm{u}_{\mathrm{el}}$, a linear decrease of structure stiffness is assumed to occur with an increase of the $u_{\max }$ value. The displacement $u$, at which the rigidity of the system reaches 0 , is called conditional destruction displacement. The displacement $u$ of the time at which the reaction of the system reaches its maximum value should be considered the actual displacement at which the structure is destroyed. Dependencies $\mathrm{R}(\mathrm{u})$ and $\gamma(\mathrm{U})$ and the corresponding "force-displacement" diagram are shown in Fig.4 


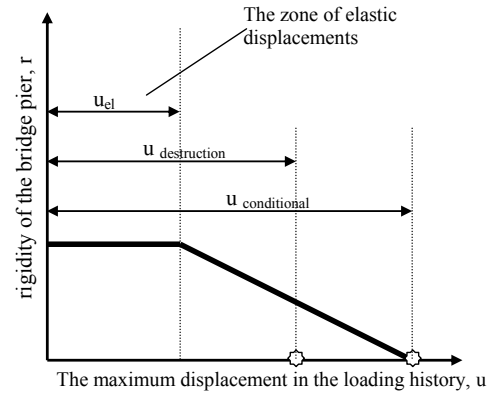

Fig. 2. Dependence of the rigidity of the bridge pier on the maximum displacement of the bridge pier in the loading history

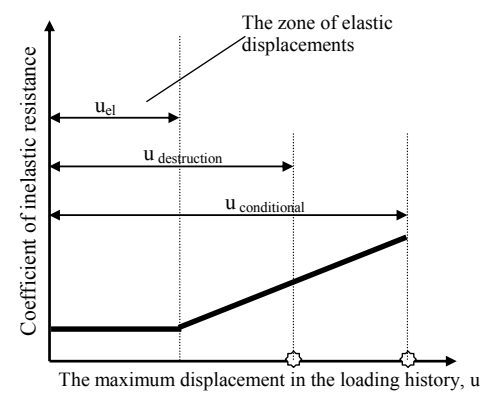

Fig. 3. Dependence of the inelastic resistance coefficient of the bridge pier on the maximum displacement of the bridge pier in the loading history

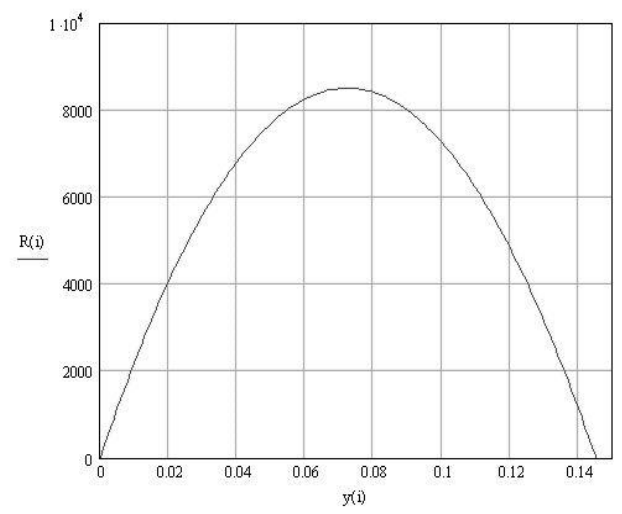

Fig.4. The system deformation curve R(u)

The oscillations of the system with degrading rigidity are described by the equation:

$$
\mathbf{M} \ddot{\mathbf{U}}+\mathbf{B} \dot{\mathbf{U}}+\mathbf{R}(\mathbf{U})=-\mathbf{M} \ddot{y}_{0}(t),
$$

where $\mathbf{U}, \dot{\mathbf{U}}, \ddot{\mathbf{U}}$ are, the displacement, velocity and acceleration of the structure respectively;

$\mathbf{M}$ is inertia matrix; $\mathbf{B}$ is damping matrix; $\mathbf{R}$ is the stiffness matrix;

$\ddot{y}_{0}(t)$ is the accelerogram of base oscillations.

The integration of the motion equation was carried out using the Duhamel integral after the expanding of the motion equations according to oscillation modes. Such expansion is carried out for each integration step. If the characteristics of the system have changed within this step, then the parameters of the motion expansion in the oscillation modes are to be change in accordance with the change in the system rigidity and damping. In this case, the exact expansion of the motion equations, i.e. taking into account the effect of damping on the oscillation modes, and the approximate expansion in terms of the vibration modes of the undamped system are possible.

\section{Result}


Let us consider an example of behavior of the structure with the MD in the case of the system degrading rigidity. The criterion of the MD effectiveness in this case may be the structure damage. Calculations show that using the MD can significantly reduce damages. Fig. 5 shows the results of calculating of a system without the MD and a system with the MD for the action of the earthquake that occurred in Ashgabat in 1948. The figure shows the initial accelerogram and the time history of damage accumulation for structures without the MD and with the MD, with initial eigen oscillation period of the construction without the MD being equal to 1.19 seconds. In this case the use of the MD reduced the damageability from $76 \%$ to $51 \%$.

In addition to calculations using accelerograms of earthquakes, synthetic accelerograms that were dangerous for the structure were generated. For this purpose, the technique described in [11] was used. In accordance with this technique, the accelerogram is specified by a certain time history process with a set of undetermined parameters that are selected to ensure proximity of the design action parameters to the actual data. In this case, kinematic, spectral and energy parameters of earthquakes are considered. Among the kinematic parameters there are, in particular, PGA and the harmonic factor

$$
\kappa=\frac{y_{0}^{(\max )} \cdot \ddot{y}_{0}^{(\max )}}{\left(\dot{y}_{0}^{(\max )}\right)^{2}}
$$

a)

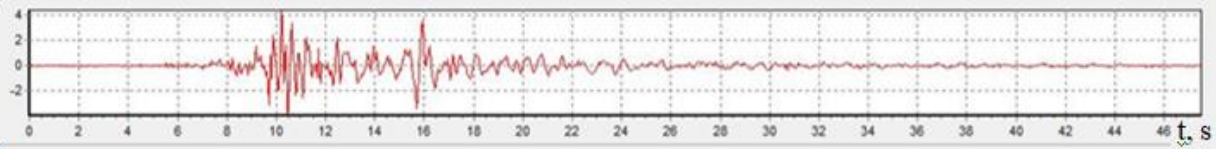

b)

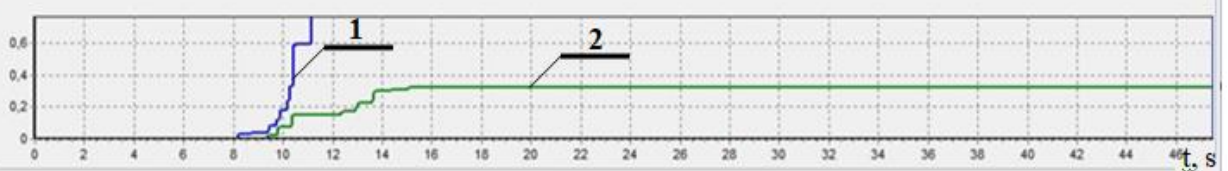

c)

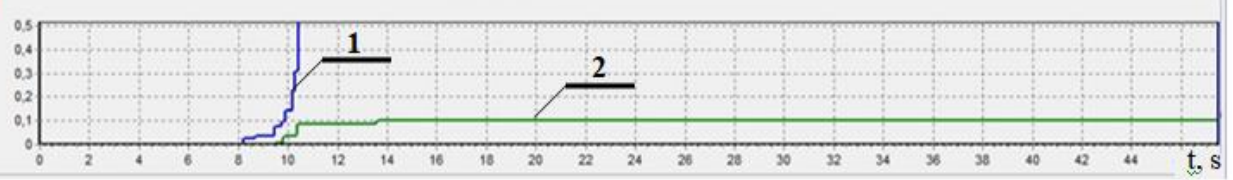

Fig. 5. The calculation results of the structure with the MD under the action of the Ashgabat earthquake.

a) accelerogram of the earthquake in Ashgabat, b) time history damage accumulation for an unprotected structure c) time history damage accumulation for the structure protected by the MD

The line No 1 describes damages at the bottom of the structure

The green No 2 describes damages in the middle point of the structure

Among the energy characteristics are the Arias intensity $\left(\mathrm{I}_{\mathrm{A}}\right)$, absolute cumulative velocity (CAV) and seismic energy density (SED), which are determined by the formulas:

$$
I_{A}=\frac{\pi}{2 g} \int_{0}^{T} \ddot{y}_{0}^{2} d t \quad C A V=\int_{0}^{T}\left|\ddot{y}_{0}\right| d t \quad S E D=\int_{0}^{T} \dot{y}_{0}^{2} d t .
$$

The spectral characteristics (the spectral action composition) are chosen as dangerous for the structure 
For the elastic system, two generated accelerograms are used to calculate the MD, which determine strains in the construction and in the MD spring [12]. These accelerograms are tuned to the first and second eigen oscillation frequencies of the system with the MD. In our case, the system does not have the fixed eigen frequency. Due to the accumulation of damages the period of structure oscillation increases from $0.464 \mathrm{~s}$ to $1.19 \mathrm{~s}$ by the time when the structure is close to collapse. The synthetic accelerograms corresponding to these periods are shown in Fig. 6
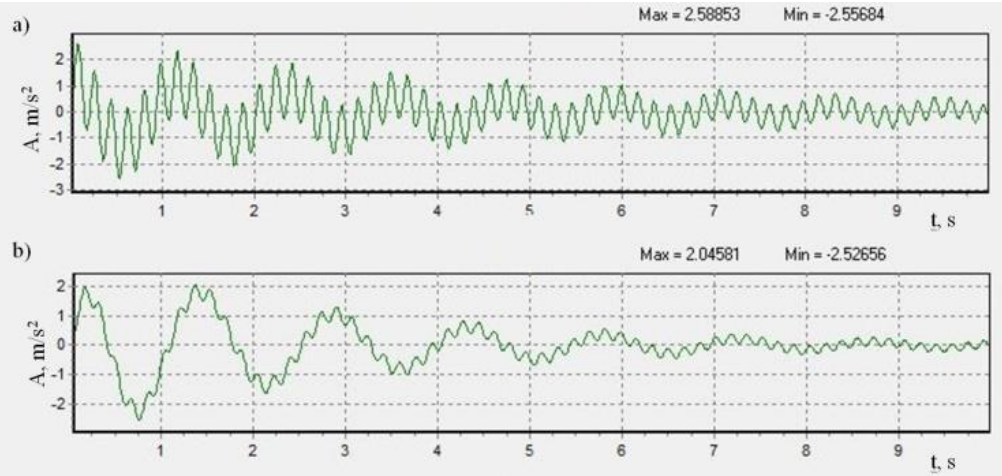

Fig.6. Accelerograms of the earthquake, generated at the resonant frequency of the structure at the elastic stage of behavior (a) and with a changed spectral composition (b)

The calculation results are shown in Fig.7. As can be seen from the picture, the accelerogram generated on the spectral composition of the damaged structure destroys the structure by $40 \%$, while the accelerogram generated on the initial resonant frequency of the elastic system leads to its destruction of up to $87 \%$.

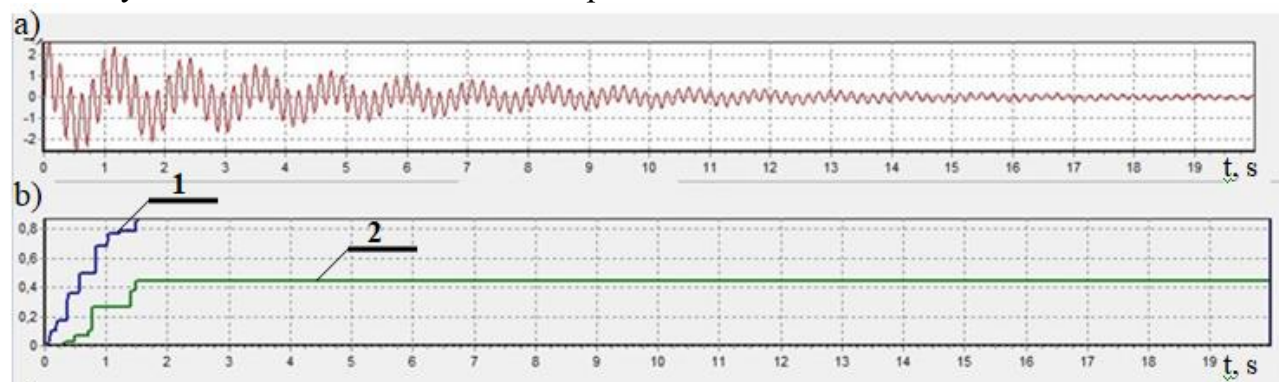

c)
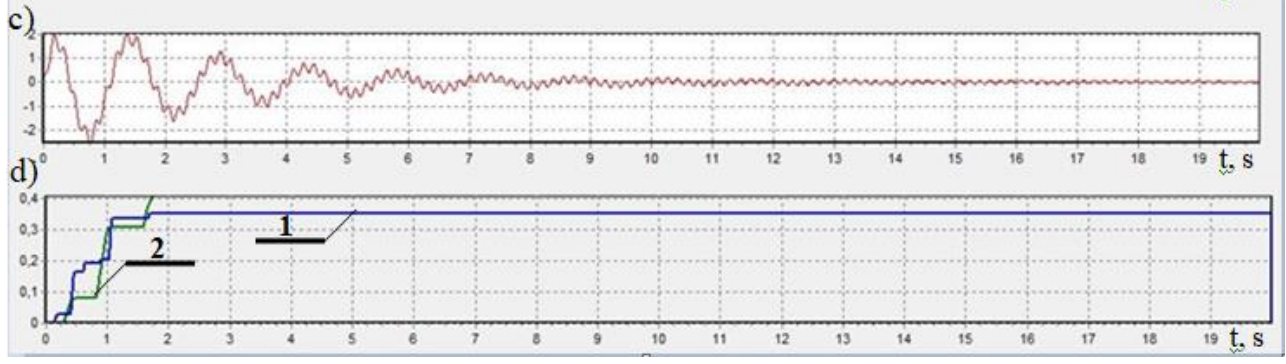

Fig.7. The results of calculating the structure with the MD for the action generated on the resonant structure frequency at the elastic stage of its behavior and with a changed spectral composition a) - Accelerogram generated on the resonant structure frequency at the elastic stage of behavior, b) time history damage accumulation for a structure with the MD and the accelerogram (a), 
c) an accelerogram generated with a modified spectral composition, d) time history damage accumulation for a structure with the MD and the accelerogram (c)

The line No 1 describes damages at the bottom of the structure

The green No 2 describes damages in the middle point of the structure

In the authors opinion it is best to keep the tuning for an elastic system as in this case the MD will suppress the resonant oscillations of the structure that arise within 5-7 seconds of an intense action. When damage begins to occur in the system, its eigen period begins to increase. But the time of the existence of a system with a fixed period is too short for resonance oscillations to be realized. Also, to make the MD operation more efficient under strong earthquakes, it is advisable to install two MDs, one of which is tuned to the natural oscillation frequency of the elastic system, and the other to a certain frequency of the system with damages.

Next, the behavior of a structure with the MD for an elastoplastic system is considered. Just as in the case of systems with degrading stiffness for an elastoplastic system, one should expect a decrease of the MD efficiency, since with the growth of plastic deformations, the eigen period of system oscillations will change. However, when a plastic deformation occurs, the MD behavior differs significantly from the above-discussed behavior of systems with degrading rigidity. Elastoplastic systems can be characterized by the AFC, although the parameters of the AFC depend on the oscillation amplitude. Using the AFC, one can select the most dangerous period of synthetic actions and a method of selecting is given in [12]. A simpler method for specifying the most dangerous frequency using the maximum on plastic deformation forces spectrum proposed by the authors is given above. To illustrate the operation of the MD in an elastoplastic system harmonic excitation was used for clarity.

Fig. 8 shows the results of calculating the system with an eigen frequency of $13.53 \mathrm{~s}^{-1}$ for a resonant sinusoid with an amplitude of $2 \mathrm{~m} / \mathrm{s}^{2}$. As can be seen from the figure, the criterion for the efficiency of the MD is the residence time in plastic state (state " 0 ") and the amplitude of plastic deformations. In the final analysis these parameters determine the work of plastic deformation, which in its turn determines the possibility of progressive collapse or low cycle fatigue of the structure.

a)

Input accelerogram

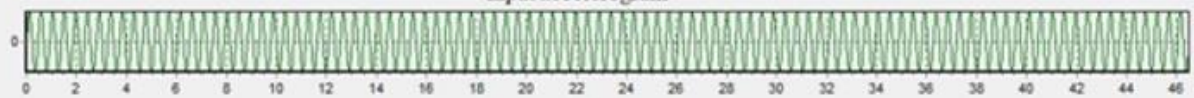

b)

Diagram of System State Interleaving

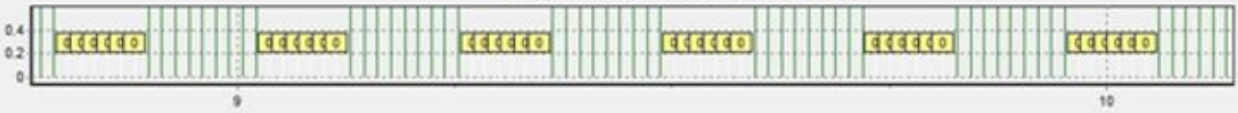

c)

Diagram of System State Interleaving

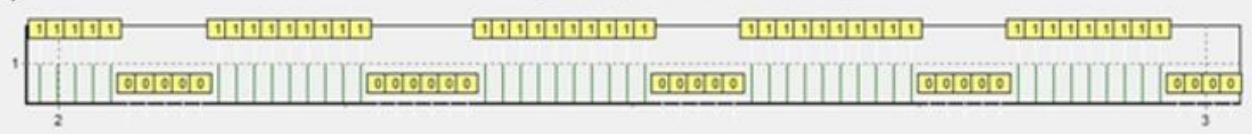

Fig.8. Calculation results of the elastoplastic system for harmonic action a) - the initial action of the resonant sinusoid

b) - the diagram of the state alternations of the unprotected system

c) - the diagram of the state alternations of the system with the MD State "0" - plastic stage of work, the state "1" - the elastic stage of work 
Since the frequency of natural oscillations varies in the oscillation process it seems reasonable to install two MDs just as for systems with degrading rigidity. The first the MD reduces the load on structures in the elastic area of the structure behavior and prevents the transition to plastic region, and the second the MD is put into operation in plastic deformation area of the structure.

\section{Conclusions}

1. The results obtained allow us to conclude, that the MD increases the construction seismic stability under destructive earthquakes

2. The MD decreases the crack propagation for systems with degrading rigidity and the work of plastic deformation forces for elastoplastic systems

3. For systems with degrading rigidity, it is advisable to tune the MD to the eigen frequency of the undamaged system

4. For elastoplastic systems, it is necessary to tune the MD to the dangerous frequency according to the gain-frequency characteristic of the system or to the maximum on the spectrum of the plastic deformation force work.

5. It seems useful to increase the structure seismic stability against strong earthquakes using two MDs, one of which is tuned to the frequency of the intact system, and the other to the frequency of the damaged system.

\section{References}

1. B.G. Korenev and L.M. Reznikov, Tuned mass dampers (Nauka, Moscow, 1988)

2. V.S. Polyakov, Construction mechanics and structure calculation 5, 49-53 (1980)

3. A.A. Nikitin, M.Yu. Tsibarova, A.M. Uzdin, "To the question of the application of large-scale tuned mass dampers for power structures," in Materials of conferences and meetings on hydraulic engineering: Improving the reliability of power structures under dynamic impacts, (Energoatomizdat, Leningrad, 1989), pp. 242-245.

4. Y. Hori, H. Kurino, Y. Kurokawa, International Journal of High-Rise Buildings 5:3, 167-176 (2016)

5. A. Shayeghi, H. Shayeghi, H. Eimani Kalasar, International Journal of Intelligent Systems and Technologies 4:1, 28-35 (2009)

6. A. Tamboli, L. Joseph, U. Vadnere, and X. Xu, "Tall Buildings: Sustainable Design Opportunities", in Council on Tall Buildings and Urban Habitat, 8th World Conference, (Dubai, 2008)

7. Alex Y. Tuan, G.Q. Shang, Journal of Applied Science and Engineering, 17:2, 141156, (2014)

8. A.M. Uzdin, S.V. Elizarov and T.A. Belash, Earthquake resistant design of transport buildings and structures (Publishing house FGOU "Training Center for Education in railway transport", 2012)

9. A.M. Uzdin, O.P. Nesterova, S.V. Prokopovich, A.A. Dolgaya, Zhang Yuan, Guan Yuhai and Wan Haibin, Russian-Chinese scientific journal "Commonwealth", 20:1, 59-66, (2017)

10. A.M. Uzdin and T.A. Sandovich, Al-Nasser-Mohomad Samih Amin. Fundamentals of the seismic resistance theory and earthquakegineering of buildings and structures (Ed. VNIIG, S.Peterburg, 1993) 
11. Recommendations on the seismic input setting for the calculation of buildings of different degrees of responsibility (KamTsentr, St. Petersburg - PetropavlovskKamchatsky, 1996)

12. A.A. Dolgaya, A.V. Indeykin, A.M. Uzdin, Theory of dissipative systems (PGUPS, S.Peterburg, 1999) 